\title{
Key mendelian variants
}

Genetic comparison of the effects of mutant and wild-type alleles is a powerful way to define gene function. But those few disease-causing variants that provide qualitatively different insights into the disease mechanisms of more common sporadic diseases have the greatest translational value.

W hile the discovery of disease-causing rare variants is becoming progressively cheaper and easier, limited only by the ability to identify and recruit affected families, the mechanistic follow-up required to understand the molecular mechanisms of disease and bring this understanding to the development of therapies is becoming progressively more expensive and complex with the advent of techniques for gene editing and sophisticated cell and animal models. Systematic sequencing (Nat. Genet 47, 717-726, 2015) is now a good way to identify a large proportion of the disease-associated variants that are not identified by the 55,009 existing genetic tests for 5,285 genes in 4,464 disorders (http://www.genetests.org/; as of 13 November 2015). For families with genetic disorders, a diagnosis is an outcome, ending their diagnostic odyssey and providing some guidance for disease management and reproductive decisions.

But which variants will provide the greatest insights into the pathogenesis of more common diseases? On page 1378, Langston et al. propose prioritizing for induced pluripotent stem cell models and gene-environment studies those alleles of mendelian subtypes of Lewy body disease that produce pathology that most closely resembles the molecular and anatomical pathology of sporadic Parkinson's disease. A corollary of this approach is that the mechanistic insights from other mendelian parkinsonian disorders, although perhaps valuable in understanding the vulnerability of dopaminergic neurons, may be poor models for the systemic and progressive molecular pathology of the sporadic disease. We welcome this Perspective as a challenge to the genetics community to focus first on the central pathogenic mechanisms of each disease process that the key mendelian variants have identified, reserving the symptomatic heterogeneity of sensitive organs damaged by mutations for future research.

Langston et al. do, however, admit that there are limitations to their prioritization of loci rather than individual variants by molecular pathology. And there are even exceptions when individual variants are selected. For example, they discuss a single variant of LRRK2 (NM_198578.3), c.6055G >A (p.Gly2019Ser), that can cause either neurodegeneration of dopaminergic neurons without Lewy body pathology or multisystem Lewy body disease in different individuals.

Up to this point, the effort in discovering all the heritable variants in the human genome that cause disease has been a matter of naming and claiming, as well as understanding mechanisms, wherever that has been possible. We agree with Langston et al. that labeling genes for particular symptom complexes should cease. The journal regards gene names as arbitrary labels rather than priority claims or indications of gene function, as molecular definitions of function (Nat. Genet. 36, 559-564, 2004) change when new molecular interactions are discovered.

At the same time, we welcome analytical and systems biology approaches that can take into account the greater locus heterogeneity of related disorders and that scale better than cell models do. When scalable technologies for functional analysis become available, it may be necessary to take the variation of the entire genome into account in every disease, but for the moment it does seem like a good idea to minimize the noise by focusing analysis of genetic mechanisms of disease on proximal pathological molecular and cellular processes before distal (and possibly convergent) symptoms.

The journal's standard for selecting informative mendelian variant studies remains the same (see previous Editorials, Nat. Genet. 46, 1153, 2014 and Nat. Genet. 45, 1411, 2013). 